\title{
Perception of induced dyspnea in fibromyalgia and chronic fatigue syndrome
}

Maaike Van Den Houte ${ }^{\mathrm{a}}, \mathrm{PhD}$, Katleen Bogaerts ${ }^{\mathrm{a}, \mathrm{b}}, \mathrm{PhD}$, Ilse Van Diest ${ }^{\mathrm{a}}$, PhD, Jozef De Bie ${ }^{\mathrm{c}}, \mathrm{MD}$, Philippe Persoons ${ }^{\mathrm{d}}, \mathrm{MD}, \mathrm{PhD}$, Lukas Van Oudenhove ${ }^{\mathrm{e}}, \mathrm{MD}, \mathrm{PhD}, \&$ Omer Van den Bergh ${ }^{\mathrm{a}}, \mathrm{PhD}$

${ }^{a}$ Health Psychology, University of Leuven, Belgium

b REVAL - Rehabilitation Research Center, Faculty of Medicine and Life Sciences, Hasselt University, Diepenbeek, Belgium

${ }^{\mathrm{c}}$ Centre for Translational Psychological Research (TRACE), Hospital ZOL Limburg, Genk, Belgium

${ }^{\mathrm{d}}$ Department of Psychiatry, University Hospital Gasthuisberg, Leuven, Belgium

${ }^{\mathrm{e}}$ Laboratory for Brain-Gut Axis Studies (LaBGAS), Translational Research Center for Gastrointestinal Disorders (TARGID), University of Leuven, Belgium.

Running head: perception of induced dyspnea in FSS patients

Key words: fibromyalgia, chronic fatigue syndrome, symptom perception, predictive coding, functional somatic syndromes

\section{Corresponding author:}

Omer Van den Bergh, PhD

KU Leuven - University of Leuven

Tiensestraat $102-3000$ Leuven, Belgium

Tel.: +32 163260 58, Fax: +3216326144

e-mail: omer.vandenbergh@ppw.kuleuven.be

Maaike Van Den Houte, <maaike.vandenhoute@kuleuven.be>

Katleen Bogaerts, <katleen.bogaerts@kuleuven.be>

Ilse Van Diest, <ilse.vandiest@kuleuven.be>

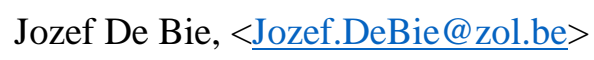

Philippe Persoons, <philippe.persoons@ kuleuven.be>

Lukas Van Oudenhove, <lukas.vanoudenhove@kuleuven.be> 


\section{Abstract}

Objective: Dyspnea perception is distorted in patients with medically unexplained dyspnea. The goals of this study were 1) to replicate these results in patients with fibromyalgia and/or chronic fatigue syndrome (CFS), and 2) to investigate predictors of distorted symptom perception within the patient group, with a focus on negative affectivity (NA), psychiatric comorbidity and somatic symptom severity.

Methods: Seventy-three patients diagnosed with fibromyalgia and/or CFS and 38 healthy controls (HC) completed a rebreathing paradigm, consisting of a baseline (60s of room air), a rebreathing phase (150s, gradually increasing ventilation, partial pressure of $\mathrm{CO}_{2}$ in the blood, and self-reported dyspnea), and a recovery phase (150s of room air). Dyspnea, respiratory flow and $\mathrm{FetCO}_{2}$ levels were measured continuously.

Results: Patients reported more dyspnea than HC in the recovery phase $(p=0.039)$, but no differences between patients and HC were found in the baseline $(\mathrm{p}=0.07)$ or rebreathing phase $(\mathrm{p}=0.17)$. No significant differences between patients and $\mathrm{HC}$ were found in physiological reactivity. Within the patient group, the effect in the recovery phase was predicted by somatic symptom severity $(p=0.046)$, but not by negative affectivity or by the number of psychiatric comorbidities.

Conclusion: This study extended earlier findings in patients with medically unexplained dyspnea to patients with fibromyalgia and CFS. This suggests that altered symptom perception is a non-symptomspecific mechanism underlying functional somatic syndromes in general, particularly in patients with high levels of somatic symptom severity. The results are discussed in a predictive coding framework of symptom perception.

Key words: functional somatic syndromes, fibromyalgia, chronic fatigue syndrome, symptom perception, predictive coding 


\section{$\underline{\text { Introduction }}$}

About $40-49 \%$ of patients in primary care present with medically unexplained symptoms (MUS; symptoms not corresponding to bodily dysfunction; 1). Some patients present with chronic MUS that are highly debilitating. Depending on the reported symptoms and the consulted medical specialty, different labels are used to describe the condition, like fibromyalgia, chronic fatigue syndrome (CFS), or medically unexplained dyspnea. Combined, these types of syndromes are referred to as functional somatic syndromes (FSS).

It has been proposed that FSS are at least partly a disorder of symptom perception (2,3). Symptom perception is influenced by (bottom-up) somatic sensations and (top-down) attentional, affective and memory processes (4). Because the relative contribution of these processes to the actual symptoms varies between and within persons, so does the correspondence between afferent input and reported symptoms. It has been proposed that FSS represent one extreme end of this continuum: afferent input is processed in such a way that eventually there is little correspondence between the afferent input and the subjective experience of symptoms $(3,5)$.

Critical variables moderating the within-person correspondence between induced physiological changes and symptom reports have been demonstrated in experimental studies using a rebreathing paradigm. In these experiments, participants breathed through a circuitry either connected to room air or to a bag initially filled with $5 \% \mathrm{CO}_{2}$ and $95 \%$ oxygen, causing gradual increase in ventilation, partial (arterial) $\mathrm{CO}_{2}$ pressure and self-reported feelings of dyspnea (air hunger). Concealed from the participant, participants switch to room air breathing after $150 \mathrm{sec}$ of rebreathing allowing recovery. These studies have shown that healthy high habitual symptom reporters and patients with medically unexplained dyspnea show a reduced within-subject correspondence between induced physiological changes and perception thereof, compared to healthy controls (6-8). However, these differences were found only after switching to room air breathing (recovery), and not during rebreathing. In addition, this reduced "body-symptom" correspondence only emerges when participants rate "breathlessness" and not when they rate "faster/deeper breathing". This pattern of results shows that when the afferent input is weak (recovery) and the context generates anticipation of symptoms, the correspondence between self-reported symptoms and induced physiological changes drops significantly in FSS compared to HC.

So far, this paradigm has only been administered in FSS patients with medically unexplained dyspnea (8). Given the debate on the specificity of different FSS (9-11) and the hypothesis that the deficit in symptom perception underlies FSS in general (3), the first goal of this study was to investigate whether results found in patients with medically unexplained dyspnea (8) extend to patients with fibromyalgia and CFS. The second goal of this study was to look for predictors of distorted symptom perception within the patient group. We therefore chose to investigate the effects of three variables 
that are related to symptom reporting, symptom severity and quality of life within the fibromyalgia/CFS patient group: negative affectivity (NA), psychiatric comorbidity and somatic symptom severity (12-16). Somatic symptom severity was measured with the somatic symptom subscale of the Patient Health Questionnaire (17).

\section{$\underline{\text { Methods }}$}

\section{Participants}

Patients were recruited through the Psychiatry Departments of East Limburg Hospital (Genk) and University Hospital Gasthuisberg (Leuven), and through a Rheumatology Center (Genk). Only patients with a doctor-based diagnosis for CFS and/or fibromyalgia were included. After inclusion, participants aditionally filled out a questionnaire checking the 1994 CDC criteria of CFS (18) and 2010 ACR criteria for fibromyalgia (19). Exclusion criteria for patients were a body mass index > 35, pregnancy, alcohol- or drug dependence, anorexia or bulimia nervosa, (history of) psychosis and chronic cardiovascular, respiratory or neurological disorders. Healthy controls (HC) were recruited through local advertisement. HC were excluded if they had any chronic medical disorders or (history of) psychiatric disorders. In order to investigate predictors within the patient group, we recruited twice as many patients as HC. HC were recruited by means of frequency matching, so that the distribution of age and gender was similar in both groups. All participants provided written informed consent. The study was approved by the Medical Ethics Committees of University Hospital Gasthuisberg, Leuven and East Limburg Hospital, Genk.

\section{Design}

This study was part of a larger study involving four experimental paradigms administered to the same participants, aiming to investigate symptom perception in fibromyalgia and CFS. Participants went through a psychiatric diagnostic interview by telephone, filled out an online questionnaire battery and participated in a single test session in either the University Hospital of Leuven or in Hospital ZOL. This test session consisted of 1) a non-invasive baseline measurement of physiological parameters, 2) a picture viewing paradigm, in which patients viewed a series of negative, positive and neutral pictures, 2) a rebreathing paradigm, 3) a conditioning paradigm with a fearful face and unpleasant sound as negative reinforcement and 4) a conditioned pain modulation paradigm in which participants received painful electrocutaneous stimulation. Only the results of the rebreathing paradigm are reported here. Detailed methods and results of the picture viewing paradigm and conditioned pain modulation paradigm are reported elsewhere $(20,21)$ 


\section{Self-report measures}

Negative affectivity was measured with the negative affect subscale of the trait Positive and Negative Affect Schedule (PANAS; 22). Respondents indicate on a five-point scale (1: very slight - 5: very much) to what extent they experience ten positive and ten negative feelings in daily life.

Somatic symptom severity was measured with the somatic symptom scale of the Patient Health Questionnaire (PHQ-15: 17). Respondents indicate to what extent they were bothered by 15 common somatic symptoms in the past two weeks on a three-point scale (0: not bothered at all - 2 : bothered a lot).

Dyspnea during the rebreathing test was measured with a 100-point numeric rating scale. Labels next to the scale were: no dyspnea (0), barely noticeable (5), very slight (10), slight (20), moderate (30), rather strong (40), strong (50), very strong (60-80), very very strong (90), unbearable (100). Dyspnea ratings were measured continuously, sampled at $10 \mathrm{~Hz}$ and stored on a personal computer.

Psychiatric comorbidity was assessed with the MINI International Neuropsychiatric Interview $(23,24)$, which is based on the DSM-IV criteria for psychiatric disorders and checks, among others, for the presence of a depressive episode, (hypo)mania, panic disorder, agoraphobia, social phobia, obsessive-compulsive disorder, post-traumatic stress disorder, generalized anxiety disorder and somatization disorder. A psychiatric comorbidity score was made and patients were allocated to one of four categories (no, 1, 2 and 3 or more comorbid psychiatric disorders).

\section{Apparatuses and physiological recordings}

The standard rebreathing paradigm (25) was used. Participants wore a nose clip and breathed through a mouthpiece. A Y-valve connected the mouthpiece to either room air or the rebreathing bag filled with a gas mixture consisting of $95 \%$ oxygen and $5 \%$ carbon dioxide $\left(\mathrm{CO}_{2}\right)$. The experimenter could switch the breathing circuit to one of the two arms of the Y-valve. Breathing through the rebreathing bag causes a progressive increase of $\mathrm{CO}_{2}$ levels in the blood, self-reported dyspnea and respiratory flow. Airflow was measured with a pneumotachograph (CD15, Validyne, Northridge, CA in ZOL; PNT 4813, Hans Rudolph, Shawnee, KA in Leuven). Fractional end-tidal $\mathrm{CO}_{2}\left(\mathrm{FetCO}_{2}\right)$ was measured with a capnograph (POET RC, Criticare Systems Inc., Waukesha, WI in ZOL; Capnogard, Novametrix, Wallingford, CT, USA in Leuven). FetCO 2 levels and respiratory flow were visually inspected and processed breath by breath with MatLab R2015a (Mathworks inc, Massachusetts, USA). To correct for equipment differences, $\mathrm{FetCO}_{2}$ was defined as the relative change in $\mathrm{FetCO}_{2}$ compared to right before the rebreathing test. To quantify respiratory flow, inspiratory time (Ti), expiratory time (Te), inspiratory volume (Vi), and expiratory volume (Ve) were extracted for every breath. Minute Ventilation was calculated per breath with the following formula: respiratory rate (RR) $\mathrm{V} V \mathrm{t}$, with $\mathrm{RR}$ $=60 /(\mathrm{Ti}+\mathrm{Te})$ and $\mathrm{Vt}=(\mathrm{Vi}+\mathrm{Ve}) / 2$. 


\section{Procedure}

Right before the rebreathing test, respiratory parameters were measured for 30 seconds without the rating scale. The rebreathing test consisted of 60 seconds of breathing room air (= baseline phase), 150 seconds of rebreathing (= rebreathing phase), and 150 seconds of breathing room air (= recovery phase). The switch between room air and the rebreathing bag was not noticeable to participants. Selfreported dyspnea, $\mathrm{FetCO}_{2}$ levels and respiratory flow were measured continuously throughout the rebreathing test.

Statistical analyses

Differences between patients and controls: Average dyspnea ratings, $\mathrm{FetCO}_{2}$ levels and minute ventilation were calculated for every 30 seconds. Multiple mixed model analyses were performed on the phases separately, with dyspnea ratings, $\mathrm{FetCO}_{2}$ levels and minute ventilation as dependent variables in separate analyses. Group (patient versus controls) and time were used as independent variables in all analyses. To control for potential baseline differences in any of the outcome variables, dyspnea rating, $\mathrm{FetCO}_{2}$ level or minute ventilation (respectively) in the last 30 seconds of the baseline phase was added as a covariate in the analyses for the rebreathing and recovery phase.

Predictors within the patient group: Similar mixed model analyses were performed on the patient group alone. First, we looked at dyspnea ratings in the different phases, in nine mixed model analyses (one for each phase*trait combination) with dyspnea as the dependent variable, and time and the trait in question (NA, PHQ-15 score, or psychiatric comorbidity score) as independent variables in separate analyses. In case one of the traits was significantly associated with dyspnea during rebreathing or recovery, physiological measures were explored using the same method. Dyspnea rating, $\mathrm{FetCO}_{2}$ level or minute ventilation (respectively) in the last 30 seconds of the baseline phase was added as a covariate in the analyses on the rebreathing and the recovery phase.

All analyses were carried out with SAS 9.4 (SAS Institute, Cary, NC, USA).

\section{$\underline{\text { Results }}$}

\section{Sample characteristics}

Eighty-one patients and $41 \mathrm{HC}$ participated in the study. Six patients and one HC stopped the rebreathing test. Data of two patients and two HC could not be analyzed due to technical problems. The final sample consisted of 73 patients (mean age: 42.37 (SD: 12.90), 10 men) and 38 HC (mean age: 40.79 (SD: 13.74), 5 men). Detailed information on marital and working status and educational level of the participants is displayed in Appendix A (Table A.1). Respiratory flow could not be calculated for three $\mathrm{HC}$ and eight patients, and FetCO2 could not be calculated for one $\mathrm{HC}$ and ten 
patients because of technical problems. According to the fibromyalgia and CFS criteria questionnaire, 36/73 patients fulfilled both the CFS and fibromyalgia criteria, 2/73 fulfilled the CFS criteria alone, and 31/73 patients fulfilled the fibromyalgia criteria alone. Four patients did not meet the criteria for CFS or fibromyalgia according to these questionnaires, but had received a doctor-based diagnosis, so were not excluded from analysis. The diagnosis the patients received (or did not receive) from their doctors did not always concur with the diagnosis they fulfilled according to our checklist. Thus, the sample can be most correctly described as a sample of patients with fibromyalgia and/or CFS. Patients who fulfilled the criteria for fibromyalgia did not differ from patients fulfilling the criteria for both fibromyalgia and CFS with regards to negative affectivity, somatic symptom severity or number of psychiatric comorbidities. Moreover, there were no main or interaction effects of diagnosis on dyspnea ratings, minute ventilation and $\mathrm{FetCO}_{2}$ in any of the phases of the rebreathing task. Therefore, in the remainder of the analyses, the patient group was treated as a whole.

Patients had higher negative affectivity (mean patients $=27.51, \mathrm{SD}=9.00$; mean controls $=16.42$, SD $\left.=5.52 ; \mathrm{t}_{105.46}=-7.99, \mathrm{p}<.001\right)$ and somatic symptom severity (mean patients $=17.34, \mathrm{SD}=4.07$; mean $\left.\mathrm{HC}=5.34, \mathrm{SD}=4.02 ; \mathrm{t}_{108}=14.77, \mathrm{p}<.001\right)$ than HC. Sixteen patients $(22.2 \%)$ had no psychiatric comorbidities, 21 patients $(29.2 \%)$ had one psychiatric comorbidity, 21 patients $(29.2 \%)$ had two psychiatric comorbidities and 14 patients (19.4\%) had three or more psychiatric comorbidities. Detailed information on psychiatric comorbidities and medication use can be found in Appendix A (Table A.2).

Differences between patients and healthy controls in each phase of the rebreathing task

Baseline phase (Figure 1, left column): Overall, dyspnea ratings rose during the baseline phase (main effect of time, $\mathrm{F}_{1,109}=17.82, \mathrm{p}<.001$ ). Patients and $\mathrm{HC}$ did not differ significantly from each other with regards to dyspnea ratings $\left(\mathrm{F}_{1,109}=3.35, \mathrm{p}=.070\right)$. There was no time*group interaction effect for dyspnea ratings $\left(\mathrm{F}_{1,109}=2.57, \mathrm{p}=.11\right)$. Minute ventilation also rose $\left(\mathrm{F}_{1,99}=6.31, \mathrm{p}\right.$ $=.014$ ), without group-related effects (main effect of group: $\mathrm{F}_{1,99}=2.14, \mathrm{p}=.15$; time* group interaction effect: $\left.\mathrm{F}_{1,99}=0.45, \mathrm{p}=.50\right)$. Patients had lower levels of $\mathrm{FetCO}_{2}$ than $\mathrm{HC}\left(\mathrm{F}_{1,98}=5.25, \mathrm{p}=\right.$ $.024)$ which overall did not change over time $\left(\mathrm{F}_{1,98}=1.36, \mathrm{p}=.25\right)$. However, a significant time*group interaction $\left(\mathrm{F}_{1,98}=4.61, \mathrm{p}=.034\right)$ indicated that levels of $\mathrm{FetCO}_{2}$ dropped for patients $\left(\mathrm{t}_{98}=2.72, \mathrm{p}=\right.$ $.008)$, but not for controls $\left(\mathrm{t}_{98}=0.62, \mathrm{p}=.54\right)$.

Rebreathing phase (Figure 1, middle column): Overall, dyspnea ratings $\left(\mathrm{F}_{4,108}=44.16, \mathrm{p}<\right.$ $.001)$, minute ventilation $\left(\mathrm{F}_{4,99}=72.03, \mathrm{p}<.001\right)$ and $\mathrm{FetCO}_{2}$ levels $\left(\mathrm{F}_{4,97}=212.92, \mathrm{p}<.001\right)$ rose during the rebreathing phase. There was no main effect of group $\left(\mathrm{F}_{1,108}=2.91, \mathrm{p}=.17\right)$, nor a significant group*time interaction effect $\left(\mathrm{F}_{4,108}=1.17, \mathrm{p}=.33\right)$ on dyspnea ratings. There were no group-related effects for minute ventilation (main effect of group: $\mathrm{F}_{1,98}=1.83, \mathrm{p}=.18$, Cohen's $\mathrm{d}=$ 
0.19; time*group interaction effect: $\mathrm{F}_{4,98}=0.75, \mathrm{p}=.56$ ) nor for $\mathrm{FetCO}_{2}$ (main effect of group: $\mathrm{F}_{1,97}=$ $3.18, \mathrm{p}=.078 ;$ time* group interaction effect: $\left.\mathrm{F}_{4,97}=0.83, \mathrm{p}=.51\right)$.

Recovery phase (Figure 1, right column): Overall, dyspnea ratings $\left(\mathrm{F}_{4,108}=20.89, \mathrm{p}<.001\right)$, minute ventilation $\left(\mathrm{F}_{4,98}=20.89, \mathrm{p}<.001\right)$ and $\mathrm{FetCO}_{2}$ levels $\left(\mathrm{F}_{4,97}=74.44, \mathrm{p}<.001\right)$ decreased during the recovery phase. A significant main effect of group on dyspnea ratings indicated that patients experienced more dyspnea overall $\left(\mathrm{F}_{1,108}=4.38, \mathrm{p}=.039\right.$, Cohen's $\left.\mathrm{d}=0.30\right)$. The time*group interaction effect on dyspnea ratings was not significant $\left(\mathrm{F}_{4,108}=0.78, \mathrm{p}=.54\right)$. There were no grouprelated effects on minute ventilation (main effect of group: $\mathrm{F}_{1,98}=1.49, \mathrm{p}=.23$; time* group interaction effect: $\mathrm{F}_{4,98}=0.23, \mathrm{p}=.92$ ) or $\mathrm{FetCO}_{2}$ (main effect of group: $\mathrm{F}_{1,97}=1.18, \mathrm{p}=.28$; time*group interaction effect: $\mathrm{F}_{4,97}=.22, \mathrm{p}=.92$ ).

\section{Effects of individual difference variables within the patient group}

Effect of negative affectivity on dyspnea ratings: There were no significant negative affectivity-related effects on dyspnea ratings in the three phases. Therefore, the relationship between negative affectivity and physiological measurements was not investigated.

Effect of psychiatric comorbidity on dyspnea ratings: There were no significant effects of psychiatric comorbidity on dyspnea ratings in the three phases. Therefore, the relationship between psychiatric comorbidity and physiological measurements was not investigated.

Effect of somatic symptom severity on dyspnea ratings (Figure 2): There were no significant effects of PHQ-15 scores on dyspnea ratings in the baseline $\left(F_{1,70}=2.01, p=.16\right.$ for the main effect of PHQ-15, $\mathrm{F}_{1,70}=0.21, \mathrm{p}=.64$ for the PHQ-15 *time interaction effect) nor in the rebreathing phase $\left(F_{1,69}=0.11, p=.74\right.$ for the main effect of PHQ-15, $F_{4,69}=1.57, p=.19$ for the PHQ15 *time interaction effect). There was also no main effect of PHQ-15 on dyspnea ratings in the recovery phase $\left(\mathrm{F}_{1,69}=0.43 \mathrm{p}=.51\right)$, but a significant time*s PHQ-15 interaction effect $\left(\mathrm{F}_{4,69}=2.55, \mathrm{p}\right.$ $=.046$ ) revealed that patients with higher levels of somatic symptom severity recovered more slowly from rebreathing with regards to self-reported dyspnea (Figure 2, upper right cell).. There were no significant effects of somatic symptom severity on minute ventilation or $\mathrm{FetCO}_{2}$ in any of the three phases.

\section{$\underline{\text { Discussion }}$}

The aim of this study was to investigate symptom perception in a rebreathing paradigm in patients with fibromyalgia and/or CFS, by 1) comparing self-reported dyspnea in patients and $\mathrm{HC}$ and 2) investigating possible predictors of dyspnea perception within the patient group. With regards to the first goal of the study, we found that patients reported more dyspnea than $\mathrm{HC}$ when afferent input was weak (recovery), but not when afferent input was intense (rebreathing). The lack of differences in physiological responses between patients and $\mathrm{HC}$ in both phases suggests that differences in subjective 
dyspnea are caused by perceptual processes. Since we controlled for dyspnea ratings during baseline, we can assume that this difference in dyspnea ratings in the recovery phase reflects a difference in the processing of induced dyspnea as separate from pre-existing differences in dyspnea between patients and HC. These results replicate and extend findings in patients with medically unexplained dyspnea to a patient population with FSS without dyspnea as primary symptom, suggesting that this overreactivity pertains to non-focal symptoms as well. In predictive coding accounts of interoception, the perception of the internal state of the body is seen as the end-result of an unconscious inferential process: similar to exteroception, somatic input is perceived in the light of "priors" (expectations, represented as distributions of neural activity) about the cause of the input (26,27). The somatic input not predicted by the prior results in prediction errors that are propagated through the system in a hierarchical error minimization process. The relative contribution of priors and prediction errors in the eventual perception depends on their relative precision: when priors are precise (i.e., high confidence) and prediction errors are imprecise (i.e. low signal-to-noise ratio), the eventual symptom experience will reflect expectations more than actual somatic input (3). Because relative precisions are influenced by learning, contextual and personality, correspondence between afferent input and symptom perception can vary between persons and within persons in different contexts (3). It's proposed that FSS are at least partly disorders of perceptual inference. Patients with FSS typically show stronger activation of affective neural networks and lower activation of inhibitory networks in response to unpleasant somatic sensations $(28,29)$, and other evidence suggests that persons with MUS show less detailed sensory-perceptual processing of afferent input, especially when the afferent input is weak (30). This results in little precise prediction errors, while on the other hand, chronic and intense concerns about somatic symptoms in FSS may create strong and precise prior expectations. In such combination, the eventual result is that in FSS, compared to HC, symptom experiences reflect prior expectations relatively more than actual somatic input $(3,5)$. In the present study, symptom-related priors were primed by telling participants explicitly that inhaling the gas mixtures would induce symptoms, and by displaying the dyspnea rating scale. In other words, symptom-related priors will be highly precise. However, in the rebreathing phase, afferent input is strong (high signal-to-noise ratio), meaning that prediction errors will also be precise. In such condition, little difference shows up between patients and HC. Afferent input is rapidly weakening in the recovery phase (reduced signalto-noise ratio), while strong priors generated by the preceding intense symptom experience remain active. The combination of the less detailed sensory-perceptual processing (creating imprecise prediction errors) with highly precise symptom-related priors in patients causes the latter to dominate symptom perception.

With regards to the second goal of the study, we found a significant moderating effect of somatic symptom severity in everyday life on dyspnea ratings in the recovery phase. Patients with higher scores on the PHQ-15 showed the above pattern in a more pronounced way: they recovered more 
slowly after rebreathing, ending with moderate to rather strong levels of dyspnea, while no differences were found in the rebreathing phase. Physiological reactivity was not different in any phase. Because we controlled for differences in baseline dyspnea, it is unlikely that these results are due to preexisting differences in dyspnea severity between patients with varying levels of somatic symptom severity. Interestingly, the PHQ-15 measures the level of distress caused by somatic symptoms experienced in daily life and does not specifically address concerns of patients with fibromyalgia and CFS. This suggests that the processes described above are enhanced by symptom-related distress in general. It is not clear, however, whether the effects are produced by having stronger priors or by less detailed sensory-perceptual processing creating less precise prediction errors.

Interestingly negative affectivity, the disposition to experience negative emotions (31), did not predict the observed effects. Negative affectivity is related to symptom reporting in healthy individuals (32) and FSS patients have, on average, higher levels of negative affectivity (33). Individuals with high negative affectivity also show lower correspondence between respiratory physiology and respiratory symptoms (34). Therefore, we expected that patients with high levels of negative affectivity would report more dyspnea in the recovery phase than patients with low levels of negative affectivity. In the FSS population, high negative affectivity is translated in higher prevalence of mood and anxiety disorders. Therefore, we also tested whether the number of psychiatric comorbidities predicted patients' response to the rebreathing test. However, negative affectivity and psychiatric comorbidity were unrelated to perceived dyspnea and to physiological responding in all phases of the rebreathing test. These results suggest that not negative affectivity in general, but symptom-specific concerns are related to low correspondence between experienced symptoms and bodily dysfunction.

Our findings have important theoretical and clinical implications. First, patients with fibromyalgia and/or CFS subjectively responded differently from $\mathrm{HC}$ to the same afferent input, also when the induced physiological dysfunction was not personally relevant to them. In other words, we extended earlier findings on dyspnea perception in patients with medically unexplained dyspnea (8) to other FSS patient groups for whom dyspnea is not a primary complaint. This suggests that the mechanisms underlying the experience of symptoms unrelated to physiological changes are trans-diagnostic $(10,11,35)$, supporting the "lumper" view of FSS (9). However, the effect of somatic symptom severity within the patient group suggests that symptom perception might only be distorted in a subgroup of FSS patients: being more distressed and concerned about symptoms enhances the described mechanisms, either by being associated with less precise prediction errors, with stronger priors or both. Second, differences between patients and HC in self-reported dyspnea were only apparent in the recovery phase, and not during rebreathing. This is at odds with what one would expect from the central sensitization framework, referring to an enhanced responsiveness and a reduced capacity to down-regulate emotional responses to unpleasant somatosensory stimuli in FSS patients (see 36,37) for reviews). In this framework, one would expect larger differences between patients and 
HC with increasing rather than decreasing stimulus intensity. Our results support the idea that FSS reflect an altered balance in the interplay between bottom-up and top-down processes, rather than just hyper-responsiveness of the central nervous system. This interpretation provides us with some clues on how to reduce symptoms in FSS. A first treatment aim would be to reduce the confidence in symptom-related priors, for instance by targeting health rumination or health anxiety by means of psychotherapy. A second treatment aim would be to increase detailed sensory-perceptual processing (i.e. augment precision of prediction errors), for instance by "interoceptive differentiation training", in which people are taught to process the sensory-perceptual properties of sensations in a more detailed way. This is possible both with sensations that are already present (such as their own heartbeat; 38) and sensations that are experimentally induced (such as respiratory loads of different intensities; 39).

Some study limitations should be mentioned. First, many patients were using medication (antidepressants, analgesics, etc.), which may have influenced symptom perception processes. However, this is inevitable when recruiting a representative patient group. This means that ecological validity remains high, but that "construct validity" of our findings is possibly compromised. Second, it is important to keep in mind that all data were cross-sectional, not permitting conclusions about directionality, let alone causality, of the observed effects.

In summary, the current study investigated symptom perception in fibromyalgia and CFS patients. Using a rebreathing paradigm, we demonstrated that patients perceive stronger dyspnea when somatosensory input is weak, whereas no differences are found when somatosensory input is strong. This was especially the case in patients with high levels of somatic symptom severity . Differences in self-reported dyspnea could not be explained by differences in objective physiological indicators of respiratory distress. Our results suggest a critical role of the interplay between bottom-up and topdown processes in symptom perception in FSS patients creating differences with HC.

\section{Acknowledgments}

The authors would like to thank the physicians and psychologists at the Psychiatry Departments of UZ Gasthuisberg, ZOL Genk, and at the Rheumatology Center in Genk for their help with the recruitment of patients; Mathijs Franssen for his help with data processing; and Katrien Coremans, Lotte Ceuterick, and Celine Samaey for their help with data collection. This research was funded by the Center for Excellence on Generalization Research (GRIP*TT; University of Leuven grant PF/10/005) and by Asthenes, a long-term structural funding-Methusalem grant by the FWO-Vlaanderen, Flemish Government, Belgium. The study was further supported by infrastructure grants from the Herculesstichting, Belgium (AKUL/13/07 and AKUL/11/08). 


\section{References}

(1) Haller H, Cramer H, Lauche R, Dobos G (2015). Somatoform disorders and medically unexplained symptoms in primary care. Deutsches Ärzteblatt International 112, 279287.

(2) Bogaerts, K (2008). Medically unexplained symptoms: a symptom perception perspective. Doctoral thesis, University of Leuven, Leuven, Belgium.

(3) Van den Bergh O, Witthöft M, Petersen S, Brown RJ (2017). Symptoms and the body: Taking the inferential leap. Neuroscience and Biobehavioral Reviews 74, 185-203.

(4) Van den Bergh O, Bogaerts K, Van Diest I (2015). Symptom perception, awareness and interpretation. In International Encyclopedia of the Social \& Behavioral Sciences, 2nd Edition, Vol 23 (ed. JD Wright), pp. 866-872. Elsevier: Oxford.

(5) Edwards MJ, Adams RA, Brown H, Paree I, Friston KJ (2012). A Bayesian account of "hysteria". Brain 11, 3495-3512.

(6) Bogaerts K, Notebaert K, Van Diest I, Devriese S, De Peuter S, Van den Bergh O (2005). Accuracy of respiratory symptom perception in different affective contexts. Journal of Psychosomatic Research 58, 537-543.

(7) Bogaerts K, Millen A, Li W, De Peuter S, Van Diest I, Vlemincx E, Fannes S, Van den Bergh O (2008). High symptom reporters are less interoceptively accurate in a symptom-related context. Journal of Psychosomatic Research 65, 417-424.

(8) Bogaerts K, Van Eylen L, Li W, Bresseleers J, Van Diest I, De Peuter S, Stans L, Decramer M, Van den Bergh O (2010). Distorted symptom perception in patients with medically unexplained symptoms. Journal of Abnormal Psychology 119, 226-234.

(9) Wessely S, White P (2004). There is only one functional somatic syndrome. The British Journal of Psychiatry 2, 95-96.

(10) White PD (2010). Chronic fatigue syndrome: Is it one discrete syndrome or many? Implications for the " one vs . many" functional somatic syndromes debate. Journal of Psychosomatic Research 68, 455-459.

(11) Lacourt T, Houtveen J, van Doornen L (2013). "Functional somatic syndromes, one or many?” An answer by cluster analysis. Journal of Psychosomatic Research 74, 6-11.

(12) Schur EA, Afari N, Furberg H, Olarte M, Goldberg J, Sullivan PF, Buchwald D (2007). Feeling bad in more ways than one: comorbidity patterns of medically unexplained and psychiatric conditions. Journal of General Internal Medicine 22, 818821. 
(13) Cosci F, Pennato T, Bernini O, Berrocal C (2011). Psychological well-being, negative affectivity, and functional impairment in fibromyalgia., Psychotherapy and Psychosomatics 80, 256-258.

(14) Wolfe F, Brähler E, Hinz A, Häuser W (2013). Fibromyalgia prevalence, somatic symptom reporting, and the dimensionality of polysymptomatic distress: results from a survey of the general population. Arthritis care \& research $65,777-785$.

(15) Häuser W, Brähler E, Wolfe F, Henningsen P (2014). Patient Health Questionnaire 15 as a generic measure of severity in fibromyalgia syndrome: Surveys with patients of three different settings. Journal of Psychosomatic Research 76, 307-311.

(16) Bogaerts K, Rayen L, Lavrysen A, Van Diest I, Janssens T, Schruers K, Van den Bergh O (2015). Unraveling the relationship between trait negative affectivity and habitual symptom reporting. PloS one 10, e0115748.

(17) Kroenke K, Spitzer RL, Williams JBW (2002). The PHQ-15: validity of a new measure for evaluating the severity of somatic symptoms. Psychosomatic Medicine 64, $258-266$.

(18) Fukuda K, Straus S, Hickie I, Sharpe M, Dobbins J, Komaroff A (1994). The chronic fatigue syndrome: a comprehensive approach to its definition and study. Annals of Internal Medicine 121, 953-959.

(19) Wolfe F, Clauw DJ, Fitzcharles M-A, Goldenberg DL, Katz RS, Mease P, Russell AS, Russell IJ, Winfield JB, Yunus MB (2010). The American College of Rheumatology preliminary diagnostic criteria for fibromyalgia and measurement of symptom severity. Arthritis Care \& Research 62, 600-610.

(20) Van Den Houte M, Bogaerts K, Van Diest I, De Bie J, Persoons P, Van Oudenhove L, Van den Bergh, O (2017). Inducing somatic symptoms in functional syndrome patients: effects of manipulating state negative affect. Psychosomatic Medicine 79, 1000-1007.

(21) Van Den Houte M, Van Oudenhove L, Van Diest I, Bogaerts K, Persoons P, De Bie J, Van den Bergh O. Negative affectivity, depression, and resting heart rate variability $(H R V)$ as possible moderators of endogenous pain modulation in functional syndromes (manuscript submitted for publication).

(22) Watson D, Clark LA, Tellegen A (1988). Development and validation of brief measures of positive and negative affect: the PANAS scales. Journal of Personality and Social Psychology 54, 1063-1070.

(23) Sheehan DV, Lecrubier Y, Sheehan KH, Amorim P, Janavs J, Weiller E, Hergueta T, Baker R, Dunbar GC (1998). The Mini-International Neuropsychiatric Interview 
(M.I.N.I.): The development and validation of a structured diagnostic psychiatric interview for DSM-IV and ICD-10. The Journal of Clinical Psychiatry 59, 22-33.

(24) Overbeek T, Schruers K, Griez E (1999). MINI International Neuropsychiatric Interview, Dutch version 5.0.0 (DSM-IV). Maastricht: University of Maastricht.

(25) Read DJ (1967). A clinical method for assessing the ventilatory response to carbon dioxide. Australian Annals of Medicine 16, 20-32.

(26) Seth AK (2013). Interoceptive inference, emotion, and the embodied self. Trends in Cognitive Sciences 17, 565-573.

(27) Barrett LF, Simmons WK (2015). Interoceptive predictions in the brain. Nature Reviews Neuroscience 16, 419-429.

(28) Montoya P, Sitges C, Garcia-Herrera M, Izquierdo R, Truyols M, Blay N, Collado, D (2005). Abnormal affective modulation of somatosensory brain processing among patients with fibromyalgia. Psychosomatic Medicine 67, 957-963.

(29) Tillisch K, Mayer EA, Labus JS (2011). Quantitative meta-analysis identifies brain regions activated during rectal distension in irritable bowel syndrome. Gastroenterology 140, 91-100.

(30) Van den Bergh O, Walentynowicz M (2016). Accuracy and bias in retrospective symptom reporting. Current Opinion in Psychiatry, 29, 302-308.

(31) Watson D, Clark L (1984). Negative affectivity: the disposition to experience aversive emotional states. Psychological Bulletin 96, 465-490.

(32) Van Diest I, De Peuter S, Eertmans A, Bogaerts K, Victoir A, Van den Bergh O (2005). Negative affectivity and enhanced symptom reports: differentiating between symptoms in men and women. Social Science \& Medicine 61, 1835-1845.

(33) Bucourt E, Martaillé V, Mulleman D, Goupille P, Joncker-Vannier I, Huttenberger B, Reveillere C, Courtois R (2016). Comparison of the Big Five personality traits in fibromyalgia and other rheumatic diseases. Joint Bone Spine 84, 203-207.

(34) Van den Bergh O, Winters W, Devriese S, Van Diest I, Vos G, De Peuter S (2004). Accuracy of respiratory symptom perception in persons with high and low negative affectivity. Psychology \& Health 19, 213-222.

(35) Witthöft M, Hiller W, Loch N, Jasper F (2013). The latent structure of medically unexplained symptoms and its relation to functional somatic syndromes. International Journal of Behavioral Medicine 20, 172-183. 
(36) Nijs J, Meeus M, van Oosterwijck J, Ickmans K, Moorkens G, Hans G, de Clerck LS (2012). In the mind or in the brain? Scientific evidence for central sensitisation in chronic fatigue syndrome. European Journal of Clinical Investigation 42, 203-212.

(37) Bourke JH, Langford RM, White PD (2015). The common link between functional somatic syndromes may be central sensitization. Journal of Psychosomatic Research 78, $228-236$.

(38) Schaefer M, Egloff B, Gerlach AL, Witthöft M (2014). Improving heartbeat perception in patients with medically unexplained symptoms reduces symptom distress. Biological Psychology, 101, 69-76.

(39) Petersen S, Schroijen M, Mölders C, Zenker S, Van den Bergh O (2014). Categorical interoception: perceptual organization of sensations from inside. Psychological Science, 25, 1059-1066. 


\section{Figure captions}

Figure 1. Least square means of dyspnea ratings (upper row), minute ventilation (in liters per minute, middle row) and mean fractional end-tidal $\mathrm{CO}_{2}$ levels (bottom row) during the baseline (left column), rebreathing (middle column) and recovery (right column) phase of the rebreathing test, for patients and controls.. p-values refer to the main effects of group (patients vs. control) on the dependent variable. Vertical bars denote standard error of the mean.

Figure 2. Least square means of dyspnea ratings (upper row), minute ventilation (in liters per minute, middle row) and mean fractional end-tidal $\mathrm{CO}_{2}$ levels (bottom row) during the baseline (left column), rebreathing (middle column) and recovery (right column) phase of the rebreathing test, for patients with differing levels of somatic symptom severity, as measured by the PHQ-15. p-values refer to the time*PHQ-15 interaction effects. Vertical bars denote standard error of the mean. 
Appendix A: Demographic information

\begin{tabular}{lcc}
\hline & Patients & HC \\
\hline Marital status (\% of participants) & & \\
\hline Single & $11 \%$ & $18 \%$ \\
Committed relationship (unmarried) & $33 \%$ & $24 \%$ \\
Married & $42 \%$ & $42 \%$ \\
Divorced & $14 \%$ & $16 \%$ \\
\hline Children (\% of participants with children) & $63 \%$ & $63 \%$ \\
\hline Working status (\% of participants) & & \\
\hline Incapacitated to work & $57 \%$ & $0 \%$ \\
Working full-time & $7 \%$ & $39 \%$ \\
Working part-time & $21 \%$ & $34 \%$ \\
Student & $3 \%$ & $8 \%$ \\
Homemaker & $1 \%$ & $5 \%$ \\
Jobseeker & $11 \%$ & $5 \%$ \\
\hline Educational level (\% of participants) & & \\
\hline Low educational level & $60 \%$ & $37 \%$ \\
High educational level & $40 \%$ & $63 \%$ \\
\hline
\end{tabular}

Table A.1. Marital status, working status, and educational level of patients and healthy controls (HC). Educational level was dichotomized into low and high, distinguishing participants who had a achieved a university or college degree from the remainder.

\begin{tabular}{lc}
\hline & $\%$ of patients \\
\hline Psychiatric comorbidities & \\
\hline Depressive episode & $44 \%$ \\
Hypomania & $0 \%$ \\
Panic disorder & $10 \%$ \\
Agoraphobia & $21 \%$ \\
Social phobia & $11 \%$ \\
Post-traumatic stress disorder & $6 \%$ \\
Obsessive-compulsive disorder & $11 \%$ \\
Generalized anxiety disorder & $55 \%$ \\
Somatization disorder & $31 \%$ \\
\hline Medication use & \\
\hline Antidepressants & $50 \%$ \\
Analgesics & $54 \%$ \\
\hline
\end{tabular}

Table A.2. Psychiatric comorbidities and medication use in the patient sample. Psychiatric comorbidity was assessed by the semi-structured MINI International Neuropsychiatric Interview. Somatization disorder was only diagnosed if the patient had multiple somatic symptoms before the age of 30 . 